\title{
La gestión por procesos un sistema de control eficiente en las empresas
}

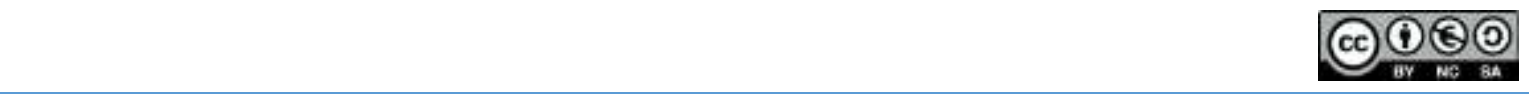 \\ Process management an efficient control system in companies
}

Germán Patricio Torres Guananga. ${ }^{1}$, Juan Carlos Rodríguez Leon. ${ }^{2}$, Alex Fabián Inca Falconi. ${ }^{3}$, Ángel Gerardo Castelo Salazar. ${ }^{4}, \&$ Emma Lucia Ríos Sanipatin. ${ }^{5}$

Recibido: 19-03-2019 / Revisado: 29-04-2019 /Aceptado: 14-05-2019/ Publicado: 14-06-2019

\begin{abstract}
.
DOI: https://doi.org/10.33262/cienciadigital.v3i2.6.600

The objective of this research was to determine if process management is an efficient control system in companies. This study was based on a qualitative approach, with a deductive process, the scope was descriptive since it details a reality, we worked with a sample of forty-four executives from six companies, the analytical and synthetic methods were combined. The results achieved have allowed us to identify the number of companies that apply the management system by processes, and of them the criteria that executives have regarding the advantages, objectives and efficiency of the model both in the management and control of companies, concluding that five of the six companies under study apply process management, and that $89 \%$ of executives surveyed believe that this model is an efficient control system in companies, which implies that this system, allows to manage the resources of a company in an efficient way, since it decreases the waste and improves the operation times, that is to say it increases the productivity and competitiveness, improvement of the quality, improves the positioning in the market, increase of income and of the profitability, therefore it is
\end{abstract}

\footnotetext{
${ }^{1}$ Escuela Superior Politécnica de Chimborazo, Facultad de Administración de Empresas, Riobamba, Ecuador, g_torres@espoch.edu.ec

2 Escuela Superior Politécnica de Chimborazo, Facultad de Administración de Empresas, Riobamba, Ecuador, juan.rodriguezl@espoch.edu.ec

${ }^{3}$ Escuela Superior Politécnica de Chimborazo, Facultad de Administración de Empresas, Riobamba, Ecuador, alex.inca@espoch.edu.ec

${ }^{4}$ Escuela Superior Politécnica de Chimborazo, Facultad de Administración de Empresas, Riobamba, Ecuador, angel.castelo@espoch.edu.ec

${ }^{5}$ Escuela Superior Politécnica de Chimborazo, Facultad de Administración de Empresas, Riobamba, Ecuador, emma.rios@espoch.edu.ec
} 
suggested to the companies analyzed to continue implementing this system and focus on the process of continuous improvement, and corresponds to the s companies that do not use this system take over it and implement process management, focused on the principle of continuous improvement.

Keywords: System, management, process, control, efficient, companies.

\section{Resumen.}

Esta investigación tuvo como objetivo determinar si la gestión por procesos es un sistema de control eficiente en las empresas. Este estudio se fundamentó en un enfoque cualitativo, con un proceso deductivo, el alcance fue descriptivo ya que se detalla una realidad, se trabajó con una muestra de cuarenta y cuatro ejecutivos de seis empresas, se combinaron los métodos analítico y sintético. Los resultados alcanzados, nos han permitido identificar el número de empresas que aplican el sistema de gestión por procesos, y de ellas el criterio que tienen los ejecutivos con respecto a las ventajas, objetivos y eficiencia del modelo tanto en la gestión y en el control de las empresas, concluyendo que cinco empresas de las seis en estudio aplican la gestión por procesos, y que el $89 \%$ de los ejecutivos encuestados creen según su experiencia que este modelo es un sistema de control eficiente en las empresas, lo que implica que este sistema, permite administrar los recursos de una empresa de manera eficiente, ya que disminuye el despilfarro y mejora los tiempos de operación, es decir aumenta la productividad y competitividad, mejora de la calidad, mejora el posicionamiento en el mercado, incremento de ingresos y de la rentabilidad, por lo tanto se sugiere a las empresas analizadas seguir implementando este sistema y enfocarse en el proceso de mejora continua, y corresponde a las empresas que no utilizan este sistema apoderarse del mismo e implementar la gestión por procesos, enfocados en el principio de mejora continua.

Palabras Claves: Sistema, gestión, proceso, control, eficiente, empresas.

\section{INTRODUCCIÓN.}

En la actualidad, el mundo se ha mostrado cambiante, con mayor competitividad, el cual exige decisiones más eficientes y eficaces a la hora de gestionar una empresa, siempre buscando que los objetivos planteados se cumplan.

Pérez Fernandez de Velasco (2004) sostiene que los procesos son conjuntos de actividades ordenadas y repetitivas, que se relacionan e interactúan entre sí, los cuales convierten entradas en salidas, agregando valor para el usuario. 
Las empresas a pesar de estar posicionadas en el mercado y contar con sus clientes establecidos, debido a la ausencia de un Sistema de Gestión por Procesos, y por ende un inadecuado control en cada uno de ellos, sufre de una deficiente gestión de sus recursos, además esto causa el incumplimiento de los objetivos empresariales, dando como resultado la insatisfacción de clientes y afectando el posicionamiento de la misma en el mercado.

Además de existir un desconocimiento de responsabilidades asignadas oficialmente del talento humano, los procesos, las funciones y la delimitación que se efectúa dentro de la unidad productiva, así como también la inexactitud que se encuentra presente en los niveles jerárquicos que originan un clima laboral inadecuado, información inoportuna y una inadecuada comunicación.

Frente a esta problemática es relevante la investigación a realizarse para determinar si la gestión por procesos es un sistema de control eficiente en las empresas, y determinar si es procedente o no la aplicación de este en las empresas.

Por lo expuesto la presente investigación pretende lograr un desarrollo empresarial en el país, en función de un Sistema de Gestión por Procesos fundamentado en el cumplimiento de los objetivos propuestos, el mismo que contribuye a optimizar los procedimientos del entorno empresarial a través de la descripción de las actividades que se realizan en la empresa, estableciéndose así un mayor control a la hora de evaluar el cumplimiento del trabajo.

\section{DESARROLLO}

\section{Sistema de Gestión}

Según Ogalla Segura, (2005). La definición propuesta por la EFQM "Es un esquema general de procesos y procedimientos que se emplea para garantizar que la organización realiza todas las tareas necesarias para alcanzar sus objetivos. El sistema de gestión es la herramienta que permite controlar los efectos económicos y no económicos de la actividad de la empresa” (pág. 1).

Según Vergara, (2009) un sistema de gestión es una herramienta que permite manejar de manera adecuada los recursos que posee una empresa, los cuales se encuentran inmersos en la elaboración de procesos necesarios para alcanzar los objetivos empresariales. Comprende las siguientes etapas:

- Etapa de Ideación. Trabajar en la idea central utilizando una metodología adecuada, logrando pulir a la idea original la cual servirá para guiar los pasos del proceso. 
- Etapa de Planeación. Planificar el objetivo a seguir y cuáles serán los pasos o estrategias que se nos llevarán a consecución de este.

- Etapa de Implementación. Es la implementación del sistema de Gestión en la empresa.

- Etapa de Control. Verifica el cumplimiento de los objetivos.

\section{La Gestión como Sistema}

Según Pacheco,Castañeda \& Caicedo, (2004). "Es la capacidad organizativa de supervivencia y proyección a largo plazo y el conjunto de instrumentos y métodos que permitan la adaptación de la organización al entorno, es una forma de regulación que se constituye en un mecanismo de orientación, control y contribuye a la vigencia de la lógica de las relaciones existentes, mediando entre los proyectos globales y los comportamientos individuales, y garantizando una estabilidad dinámica" (p. 8).

La Gestión como un Sistema, regula una serie de etapas unidas en un proceso continuo utilizando métodos, técnicas y herramientas y controlando el correcto funcionamiento de la empresa buscando una estabilidad dinámica.

\section{Indicadores de Gestión.}

Según Ogalla Segura, (2005) "Miden la actividad global del proceso de negocio (tiempo de ciclo del proceso, costes, reclamaciones de los clientes, calidad percibida, etc.). Medida operativa que utiliza la organización para supervisar, entender, predecir y mejorar los probables resultados clave del rendimiento de esta. Estará alineado con la política, estrategia y objetivos previstos" (p. 24).

Los Indicadores de Gestión son relaciones matemáticas que se expresan de forma cuantitativa, permiten evaluar el cumplimiento de los objetivos y desempeño de la empresa, entregando resultados que sirven para comparar lo ejecutado con lo planificado. Además, estos deben ser simples, adecuados, establecidos en un periodo de tiempo, incluir a los usuarios, prestar utilidad y oportunidad.

Según Consejo Nacional de Evaluación de la Política de Desarrollo Social, (2013) es importante evaluar los sistemas administrativos por procesos mediante el cuatro dimensiones generales de los indicadores de gestión como: eficacia, efectividad, economía y calidad porque permiten estandarizar el cumplimiento y rendimiento del talento humano.

- Eficacia: Miden el grado del cumplimiento de los objetivos establecidos, es decir, dan evidencia sobre el grado en que se están alcanzando los objetivos descritos. 
- Efectividad: Miden la relación entre el logro del programa y los recursos utilizados para su cumplimiento. Estos indicadores cuantifican lo que cuesta alcanzar el objetivo planteados, sin limitarlo a recursos económicos; también abarca los recursos humanos y materiales que el programa emplea para cumplir el objetivo específico.

- Economía: Miden la capacidad del programa para administrar generar o movilizar de manera adecuada los recursos financieros. Estos indicadores cuantifican el uso adecuado de estos recursos, entendiendo como la aptitud del programa para atraer recursos monetarios ajenos a él que le permitan potenciar su capacidad financiera y recuperar recursos financieros prestados.

- Calidad: Miden los atributos, las capacidades o las características que tienen o deben tener los bienes y servicios que se producen. Los programas establecen las características mínimas que han de cumplir los bienes y servicios que entrega a la población; los indicadores de calidad permiten monitorear los atributos de estos productos desde diferentes perspectivas.

Los indicadores de Gestión se construyen en base a las dimensiones y ámbitos de desempeño, los cuales deben ser medidos de acuerdo a los objetivos y a su vez cuantifican el logro de los mismos, por lo cual se consideran cuatro dimensiones generales para los indicadores como son: la eficacia (brindan información sobre el efecto deseado), eficiencia (miden la el cumplimiento adecuado de un función), calidad (miden las perspectivas de los productos o servicios entregados a clientes) y economía (miden la capacidad de administrar los recursos financieros).

\section{Estructura de los Sistemas de Gestión}

Según Fernández Ricardo, (2006). "Los Sistemas de gestión ya sea de forma individual o integrada, deben estructurarse y adaptarse al tipo y las características de cada organización, tomando especialmente en cuenta los elementos que le sean apropiados. Para ellos se debe definir claramente; La estructura organizativa, incluyendo funciones, responsabilidades, líneas de autoridad y de comunicación; los resultados que se pretende lograr; los procesos que se llevan a cabo para cumplir los fines; los procedimientos mediante los cuales se ejecuta las actividades y las tareas; y los recursos que se disponen" (p. 16).

En la estructura de los Sistemas de Gestión, se brinda información oportuna a los usuarios internos y externos de la empresa, utilizando herramientas matemáticas y de gestión que permitan lograr las metas establecidas.

\section{Gestión por Procesos.}

Según Agudelo \& Escobar, (2008). "Se realiza mediante el giro permanente del ciclo PHVA: Planear, Hacer, Verificar, y Actuar, también denominado el Círculo de Deming, que es la concepción gerencial básica para dinamizar la gerencia del día a día: relación entre las 
personas y los procesos en el trabajo diario y que se debe aplicar disciplinadamente y garantizar un resultado a través de los objetivos que en términos de calidad. Estos objetivos y su cumplimiento determinan la superación de las expectativas del cliente, lo cual se convierte, además, en el medio para lograr a la rentabilidad y finalmente la prosperidad de la organización" (p. 20-22).

La Gestión por Procesos es la transformación de la gestión clásica o tradicional, a una por procesos basados en la calidad; es un nuevo enfoque que busca que las entradas se transformen en salidas, persiguiendo el mejoramiento continuo, cuidando que las actividades realizadas de manera individual y grupal cumplan con el resultado esperado y añadan valor a los productos o servicios que serán entregados para el cliente.

\section{Características de la Gestión por Procesos}

Según Universidad Champagnat, (2002). Las características muestran como la Gestión por procesos ha evolucionado modificando a la organización sin dañar la estructura organizacional.

- Analiza las limitaciones de la organización funcional vertical para mejorar la competitividad de la empresa

- Reconoce la existencia de los procesos internos

- Identifica las necesidades del cliente externo y orientar a la Empresa hacia su satisfacción

- Entiende las diferencias de alcance entre la mejora orientada a los procesos y aquella enfocada a los departamentos a las funciones

- Asigna responsabilidades a cada proceso

- Establece en cada proceso indicadores de funcionamiento y objetivos de mejora

- Evalúa la capacidad del proceso para satisfacerlo

\section{Beneficios de la Gestión por Procesos.}

Según Agudelo \& Escobar, (2008) es beneficioso en una empresa alcanzar un mejoramiento continúo garantizando que los procesos existentes sean de calidad, también ayuda a realizar un análisis de la situación actual y establece estrategias para llegar a la situación deseada por la empresa.

- Garantiza que los procesos de la empresa sean gerenciados donde se ejecutan.

- Eliminar el trabajo innecesario, es decir, el que no genera beneficios para el cliente final, el que no agrega valor y, por tanto, el comprador o usuaria no está dispuesto a pagar por él.

- Mantiene los niveles alcanzados y mejorar.

- Elimina las causas fundamentales de los problemas. 
- Posibilita que la alta dirección disponga de más tiempo para pensar en el futuro y en el mercado de la empresa.

Según Bravo Juan, (2011). Es una disciplina de gestión que ayuda a la dirección de la empresa a identificar, representar, diseñar, formalizar, controlar, mejorar y hacer más productivos los procesos de la organización para lograr la confianza del cliente. La estrategia de la organización aporta las definiciones necesarias en un contexto de amplia participación de todos sus integrantes, donde los especialistas en proceso son facilitadores (p. 9).

La Gestión por procesos mira a la empresa como un todo y a sus empleados como una parte escencial en la consecución de las metas empresariales, dado que ellos aportan en forma directa al mejoramiento continuo en beneficio del cliente. Considerándolo como un cambio positivo y fomentando a que exista una cultura empresarial.

\section{Importancia de la Gestión por Procesos.}

Según Zaraztiegui, (1999) Afirma que los procesos se consideran actualmente como la base operativa de gran parte de las organizaciones y gradualmente se han convertido en la base estructural de un número creciente de empresas. Los procesos son posiblemente el elemento más importante y extendido en la gestión de las empresas innovadoras, especialmente de las que basan su sistema de gestión en la calidad total. Este interés por los procesos ha permitido desarrollar una serie de procesos ha permitido desarrollar una serie de técnicas relacionadas con ellos. (p.81)

Es de vital importancia que exista un adecuado control en los procesos que se realizan dentro de la empresa, asegurando que el producto o servicio final sea de calidad, por lo tanto, para que éste alcance la misma, debe seguir un conjunto de pasos, dando como resultado la mejora continua.

\section{Principios de la Gestión por Procesos}

Según Alvarado (2013), En el Registro Oficial de la Secretaria Nacional de la Administración Pública, Capítulo 2, afirma que "La administración por procesos se rige por los principios de Administración Pública establecidos en la Constitución de la República del Ecuador” (p.3$4)$.

Evaluación permanente y mejora Continua. Busca incrementar las capacidades institucionales mediante una evaluación permanente, interna y externa, orientada a la identificación de oportunidades para la mejora continua de los procesos, servicios y prestaciones públicas centrados en el servicio al ciudadano y obteniendo resultados para la adecuada rendición de cuentas. 
Enfoque en el ciudadano, beneficiario y usuario. El eje fundamental de la intervención es beneficiario o usuario de los servicios, lo cual quiere adoptar enfoques y diseñar los procesos para brindar servicios desde la perspectiva de estos actores, mejorando la ejecución de los procesos internos y su integración.

Coordinación y cooperación. Todos los órganos contribuirán a la prestación de servicios al usuario en un enfoque sistémico.

Eficiencia y optimización. La Gestión por procesos estará orientada a optimizar los resultados alcanzados por la institución, en la relación a los recursos disponibles e invertidos en su consecución.

Sostenibilidad y transparencia. Se orientara al uso de herramientas tecnológicas para automatizar los procesos optimizados, con el propósito de fomentar la transparencia, incrementar el control y mantener su sostenibilidad en el tiempo.

\section{Gestión por Procesos en la Calidad}

Según Fernandez de Velasco Jose, (2010) La Gestión por Procesos no es un modelo ni una norma de referencia sino un cuerpo de conocimientos con principios y herramientas específicas que permiten hacer realidad el concepto de que la calidad (P. 45).

La Gestión por Procesos se basa en la calidad ya que se encuentra ligada al cuarto principio "Un enfoque basado en procesos" de la gestión de la calidad.

\section{Gestión por Procesos en la Empresa}

Según Fernández de Velasco, (2010) La Gestión por Procesos está entre las prácticas más avanzadas de gestión empresarial que permite desplegar la estrategia corporativa mediante un esquema de Procesos Calve. Entendemos que un proceso merece ser caracterizado como clave cuando está directamente conectado con la estrategia corporativa, relacionado con un factor crítico para el éxito de la empresa o con alguna de sus ventajas competitivas, también se fundamenta en el trabajo en equipo, Equipo de Procesos, permitiendo hacer realidad la gestión participativa, además en la medida que los procesos son transversales, atraviesan los departamentos de la empresa, contribuyen a cohesionar la Organización y Busca la eficacia global (P.46).

Además, se debe impulsar el trabajo en equipo y el espíritu de grupo, logrando con esto que la cultura empresarial se fortalezca, para lo cual los empleados deben perseguir un objetivo común, dejando de lado los objetivos departamentales o personales en beneficio de la empresa. 


\section{Los Modelos de Gestión y el Enfoque basado en procesos.}

Beltrán, Carmona, Carrasco, Rivas y Tejedor, (2002). Es una cuestión innegable el hecho de que las organizaciones se encuentran inmersas en entornos y mercados competitivos y globalizados; entornos en los que toda organización que desee tener éxito (o, al menos, subsistir) tiene la necesidad de alcanzar "buenos resultados" empresariales. Las organizaciones necesitan gestionar sus actividades y recursos con la finalidad de orientarlos hacia la consecución de los mismos, lo que a su vez se ha derivado en la necesidad de adoptar herramientas y metodologías que permitan a las organizaciones configurar su Sistema de Gestión. (p. 9-10).

Todas las organizaciones desean alcanzar resultados positivos, pero para ello es necesario tener una adecuada gestión tanto de los procesos como de los recursos. Para adoptar este nuevo Sistema de Gestión es necesario utilizar herramientas y metodologías que ayuden a dirigir y controlar la empresa.

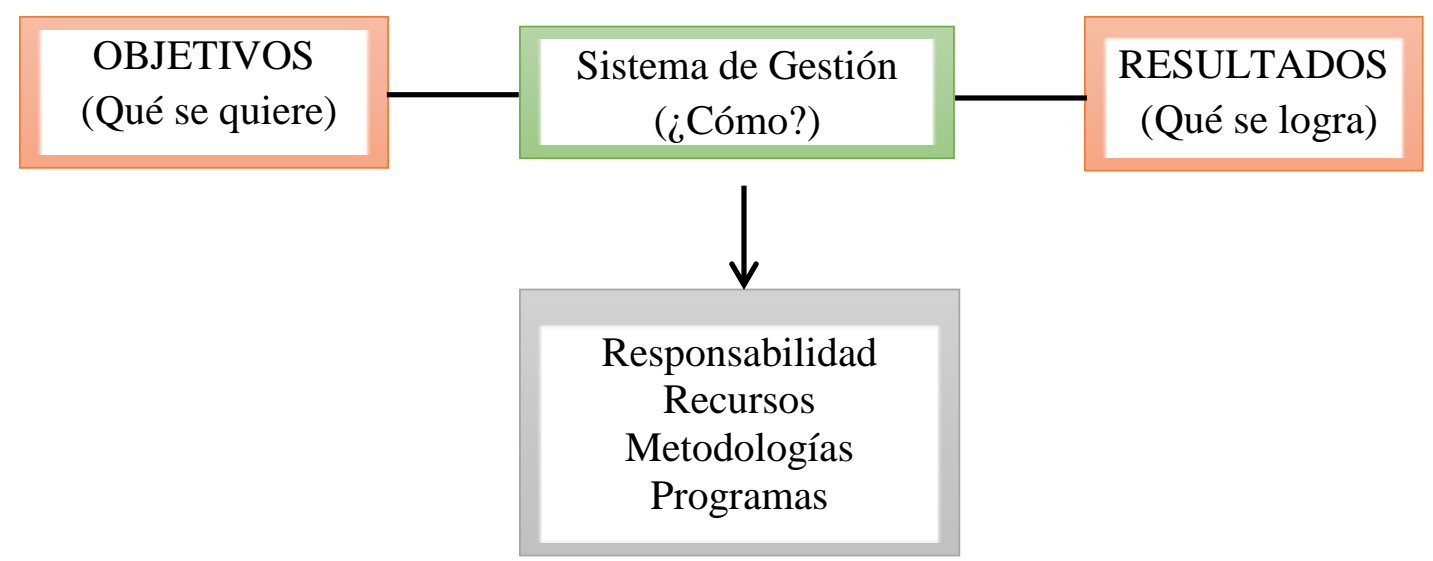

Gráfico 1. El sistema de gestión como herramienta para alcanzar los objetivos Fuente: Beltrán et al., (2002). Guía para una gestión basada en procesos

\section{Tipos de Procesos.}

Según Pérez, (2010) afirma que al no existir normalización ni práctica generalmente aceptada al respecto, vamos a distinguir los procesos por su misión; proponemos la siguiente clasificación:

Existen tres tipos de proceso y estos son; Los procesos Operativos que se encuentran inmersos dentro del proceso productivo, relacionados con las necesidades y requerimientos del cliente estos, están ligados directamente a la consecución de los objetivos empresariales; Los procesos de Apoyo brindan los recursos necesarios a los demás procesos de acuerdo a los requerimientos de los clientes; Los procesos de Gestión son los encargados de asegurar 
un control adecuados en los demás procesos y obtener la información necesaria para realizar mejoras en los mismos.

\section{Elementos de un Proceso}

Según Pérez, (2010) Todo proceso consta de los siguientes elementos:

- Un input (entrada principal), productos con unas características objetivas que responda al estándar o criterio de adaptación definido: la factura del suministrador con los datos necesarios.

- La secuencia de Actividades propiamente dicha precisa de medios u recursos con determinados requisitos para ejecutarlo siempre bien a la primera y entregar el output al siguiente eslabón del proceso administrativo.

- Un output (salida) producto con la calidad exigida por el estándar del proceso, la salida es un producto que va destinado a un usuario o cliente (externo o interno); el output final de los procesos de la cadena de valor es el input o una entrada para un proceso del cliente.

- indicadores de funcionamiento del proceso y medidas de resultados del producto del proceso y del nivel de satisfacción del usuario (interno muchas veces).

\section{Modelamiento Visual de los procesos}

Según Bravo, (2009). "El modelamiento visual de los procesos es una nueva propuesta de la teoría de los modelos para lograr la participación de todas las personas de la organización. Permite tomar conciencia de lo que hacemos y como lo hacemos. Tiene que ver con detenerse, mirar y escuchar para reflexionar y actuar" (p.38).

Se enfoca en analizar el entorno buscando la participación organizacional, además aplicar una metodología para diseñar los procesos y adecuarlos a la realidad.

\section{Mapa de Procesos}

Según Fernández, (2010). Muchos de los procesos fluyen horizontalmente a través de las clásicas organizaciones funcionales; en algún área pudiera haber un "nicho de poder". El funcionamiento de este proceso suele ser más visible desde el cliente que desde el interior de la empresa. El mapa de procesos es una herramienta que permite mostrar las interrelaciones a nivel macro. Así los procesos Operativos interactúan con los de Apoyo porque comparten "necesidades" y "recursos" y con los de Gestión comparten "datos" e "información". (p. 113)

El mapa de procesos es la representación detallada, gráfica y holística que describe, identifica y clasifica todos los procesos y actividades existentes dentro de la empresa utilizando la cadena de valor para posicionarlos de manera óptima. 


\section{Flujograma de Información}

Según Bravo, (2009). "Describe y representa una guía de las actividades del proceso, es un tipo de modelo que proporciona amplia información acerca de varios aspectos del proceso: flujo, mensajes, actividades, estructuras y tecnología. El flujo es la secuencia y temporalidad, incluye las actividades e interacciones. Los mensajes son el medio de comunicación, pueden ser documentos, comunicaciones electrónicas u orales. Las actividades son acciones que realizan cargos o roles. La estructura queda representada por columnas" (p. 377).

Un Flujograma de Información es la representación gráfica del proceso de actividades donde se muestran las situaciones y relaciones mediante símbolos, en su aplicación permite identificar oportunidades de mejora en los procesos.

\section{El Ciclo de la Gestión - PDCA}

Según Pérez, (2004). El ciclo se desencadena porque existe un Objetivo a conseguir o problema a solucionar, es esencial que el objetivo sea medible o evaluable, se desencadena la etapa de la planificación. Esta etapa concluye con la elaboración de un plan con las acciones a tomar y la determinación de los recursos disponibles. La fase de Ejecución se refiere a asegurar la implantación de las acciones previamente planificadas, no de aquello que sabemos, podemos o nos gusta hacer. Comprobación se ha de verificar, con la periodicidad definida, si las acciones implantadas han aportado los resultados esperados. Comunicar los resultados y analizar las desviaciones. Actuar podemos interpretar como revisar, optimizar, industrializar, explorar o transversalizar las acciones de mejora. (p. 111)

El ciclo PDCA (Planificar, Hacer, Verificar y Actuar) o conocido también como el círculo de la mejora continua se describe las cuatro fases que son necesarias para alcanzar la mejora continua en una empresa donde como primera Fase se haya el Planificar ( realizar un plan de actividades a realizar), segunda Fase Hacer ( realizar cambios para implantar la mejora propuesta), tercera Fase Controlar o Verificar ( revisar los resultados obtenidos del implante de mejora) y cuarta Fase Actuar ( Aprobar o reformular la mejora).

\section{Normas ISO 9001.}

Según Montaño, (2003). "Es un sistema de gestión de calidad que establece los métodos para administrar eficientemente los procesos de operación, de manera que se tenga la capacidad de producir bienes y servicios que satisfagan las necesidades de los clientes, todo ello de forma documentada que demuestre evidentemente que se cumple con estos requisitos" (p.12).

"Este sistema ha sido desarrollado por el Comité Técnico ISO/TC 176 de la Organización Internacional de normalización, que es una federación mundial de organismos nacionales de normalización. Tiene como Objetivo desarrollar un sistema de aseguramiento de calidad que permita mejorar continuamente, al corregir y prevenir los defectos" (p.13). 
Las normas ISO 9001es internacional de renombre y la más utilizada en todo el mundo, se concentra en velar por la satisfacción del cliente entregando bienes y servicios de calidad, por lo cual establece métodos para administrar procesos de manera eficiente buscando siempre la mejora continua.

\section{Normas ISO 9001:2015}

Según Traducción oficial norma internacional ISO 9001:2015, (2015), La norma ISO 9001:2015 ha establecido una estructura de Alto nivel la cual se verá favorecerá, obtendrá una disminución en los tiempos y recursos invertidos en su gestión de forma considerable. Su nueva estructura constara de 10 puntos; 1. Alcance, 2. Referencias Normativas, 3. Términos y Definiciones, 4. Contexto de la organización, 5. Liderazgo, 6. Planificación, 7. Soporte, 8. Operación, 9. Evaluación del Desempeño, y 10. Mejora.

\section{Objetivos de la norma ISO 9001:2015}

- Tomar en cuenta el impacto de la tecnología, incorporar modelos y prácticas para los negocios de actualidad.

- Generar confianza en las organizaciones certificadas mediante los requisitos de la norma.

- Crear mayor compatibilidad con empresas de servicios.

- Que la implementación de esta norma dure un tiempo de 10 años.

Las principales modificaciones fueron, que introduce un pensamiento basado en riesgos y la eficacia, es más flexible el sistema de documentación y facilita la aplicación al cualquier tipo de empresa, además incrementa el nivel de detalle en la información en procesos y controles, liderazgo y presentación de la dirección, también analiza el contexto y las partes interesadas.

\section{La mejora continua y la organización}

Una organización es una unidad viva (conjunto de personas proveedoras) que pretende sobrevivir en un determinado entorno. Para ello, a partir del análisis de este, lleva a cabo una serie de actividades (procesos) dirigidas a añadir valor a recursos propios y ajenos, transformándolos así en recursos requeridos por otras organizaciones (conjunto de personas cliente). La voluntad y capacidad de adaptarse a las necesidades de los clientes y la voluntad y capacidad de añadir valor son las bases conceptuales a partir de las cuales la mejora continua se convierte en una forma de hacer las cosas, en un estilo.

Es necesario que cada empleado conozca exactamente lo que se espera de él y cómo será evaluada su contribución a los objetivos de la organización. Las personas se han de implicar en la detección de errores y en la elaboración de estrategias de mejora. La Dirección debe ser capaz de motivar y reconocer a sus empleados. Reconocer significa comunicarles y hacerles 
saber que la organización aprecia y valora su labor y su esfuerzo. El reconocimiento es una poderosa fuerza que puede aportar a los empleados.

- Ganas de pertenecer a la organización.

- Sentimiento de grupo.

- Ganas de trabajar y de esforzarse.

- Autoestima personal y de grupo.

La mejora continua es un valor que no puede ser impuesto a los empleados, sino que tiene que salir de ellos mismos. Conseguir que los empleados puedan aportar lo mejor de sí mismos y así garantizar el éxito en la mejora continua de la organización exige gestionar tres requisitos, como muestra el siguiente gráfico.

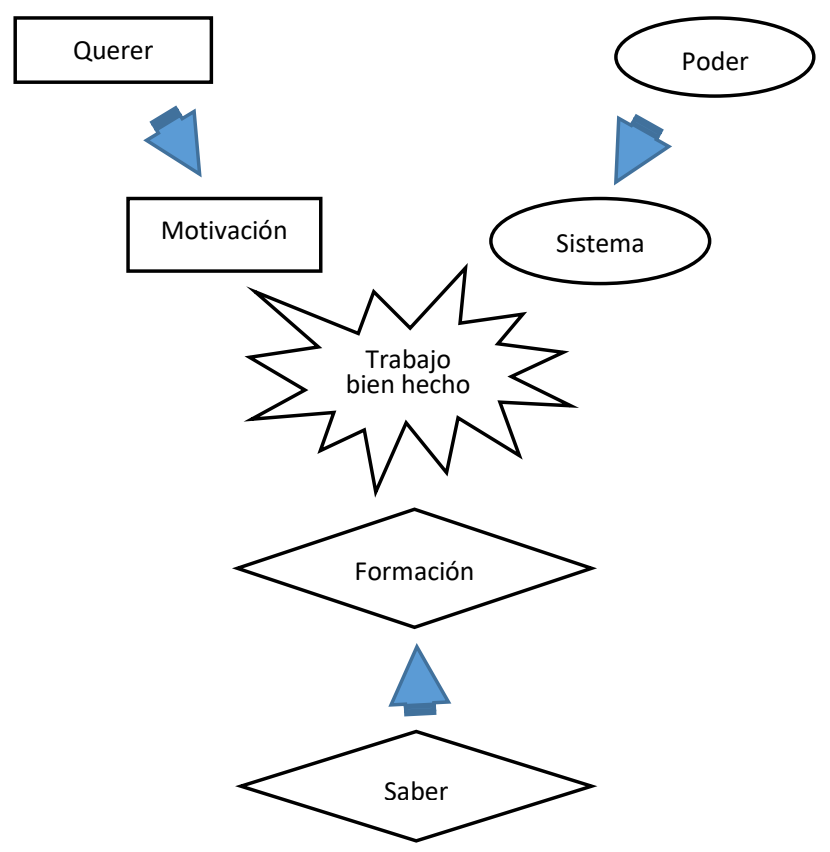

Gráfico 2. Trabajo bien hecho

Fuente: Ministerio de Fomento, España Modelos para implementar la mejora continua en la gestión de empresas

\section{Metodología}

La presente investigación dispondrá de un enfoque cualitativo porque a través de la investigación se describen los procesos de las empresas en estudio, utilizando la información recolectada, mediante entrevistas, con cuestionarios prediseñados.

Es de tipo descriptiva ya que se detalla una realidad y se realiza un análisis del porque y para que se está realizando esta investigación y describiendo paso a paso los procesos existentes en las empresas. Es documental ya que se consultó temas relacionados, se recopilo información escrita por diferentes autores para el desarrollo del Sistema de Gestión por Procesos. 
Se utiliza el Método Inductivo y deductivo ya que parte de hechos o fenómenos particulares a conclusiones generales y viceversa, se caracteriza por tener cuatro etapas; Observación, análisis, derivación y la contrastación, de igual manera, se combinaron los métodos analítico y sintético.

La población de estudio lo constituyen seis empresas de la provincia de Tungurahua, en las cuales se encuestó a los gerentes y jefes departamentales, dando un total de 44 encuestados.

\section{Resultados}

Es importante verificar la aplicación del Sistema de gestión por procesos en las empresas en estudio, para ello se realizó 44 encuestas a los ejecutivos de las diferentes empresas, obteniendo los siguientes resultados:

\section{1. ¿La empresa que usted representa y o es parte cuenta con un Sistema de Gestión por procesos (SGP) normalizado?}

\section{Su empresa cuenta con un SGP}

normalizado

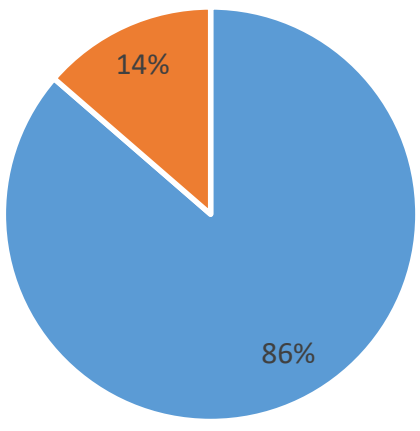

- SI

- NO

Gráfico 3. Su empresa cuenta con un (SGP) normalizado

Fuente: Elaboración propia

\section{Interpretación}

La mayoría de los ejecutivos de las empresas, específicamente el $86 \%$ indicaron que si cuentan con un Sistema de gestión por procesos, en su empresa, y tan solo el 14\% mencionan que no cuentan con este modelo de gestión. 
2. ¿Cuáles son las ventajas de implementar un sistema de gestión por procesos (SGP) en su empresa?

\section{Cuáles son las ventajas de implementar un SGP}

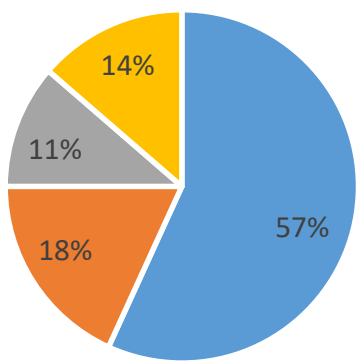

- Mejora la productividad

- Elimina la duplicidad de tareas

- Reducción de tiempos

- Mejora la rentabilidad

Gráfico 4. Cuáles son las ventajas de implementar un SGP.

Fuente: Elaboración propia

\section{Interpretación}

Del total de encuestados, el $57 \%$ manifiestan que la principal ventaja de implementar un sistema de gestión por procesos es la mejora de la productividad, mientras que el $18 \%$ manifiestan que este sistema elimina la duplicidad de tareas, y un $14 \%$ de ejecutivos cree que mejora la rentabilidad, y por último un $11 \%$ de los encuestados cree que la gestión por proceso reduce los tiempos. Por lo expuesto, la principal ventaja es la mejora de la productividad, lo que se traduce en el posicionamiento del mercado debido al mejoramiento continuo en la calidad de los procesos.

\section{3. ¿Cuál es el objetivo del Sistema de Gestión por Procesos y la mejora continua en} la empresa?

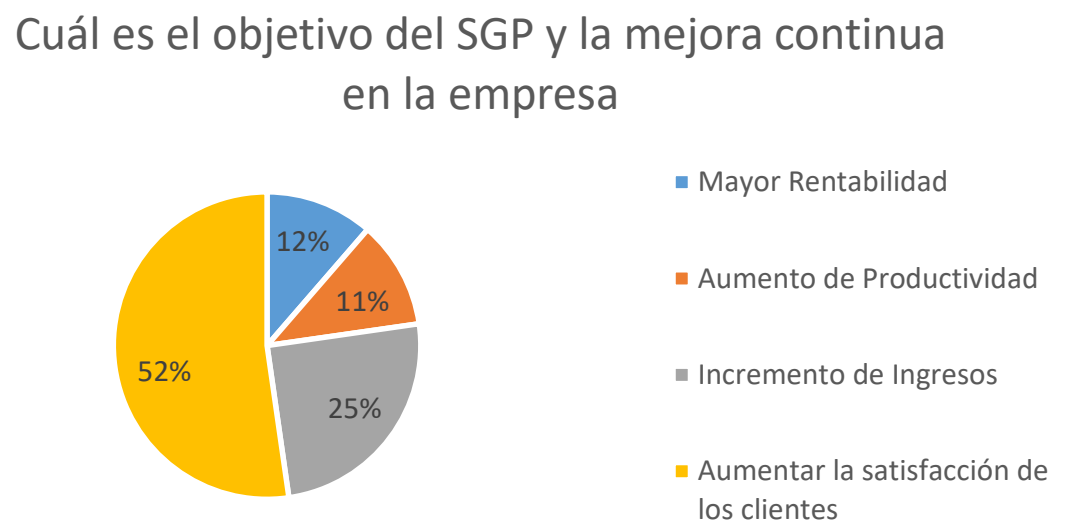

Gráfico 5. Cuál es el objetivo del SGP y la mejora continua en la empresa Fuente: Elaboración propia 


\section{Interpretación}

Los resultados en esta pregunta permiten divisar que la mayoría de los ejecutivos, el 52\% manifiestan que el principal objetivo de implementar un sistema de gestión por procesos es aumentar la satisfacción de los clientes, mientras que el $25 \%$ dicen que el objetivo de este sistema es el incremento de los ingresos, de igual manera, el $12 \%$ de ejecutivos creen que aumenta la rentabilidad, y por último un $11 \%$ de los encuestados cree que el objetivo de la gestión por procesos es el aumento de la productividad. Por lo expuesto, el objetivo de implementar un sistema de gestión por procesos es aumentar la satisfacción de los clientes, lo que se traduce en el posicionamiento del mercado, aumento de los ingresos, y mejora de la rentabilidad.

\section{4. ¿La gestión por procesos es una herramienta eficiente de gestión?}

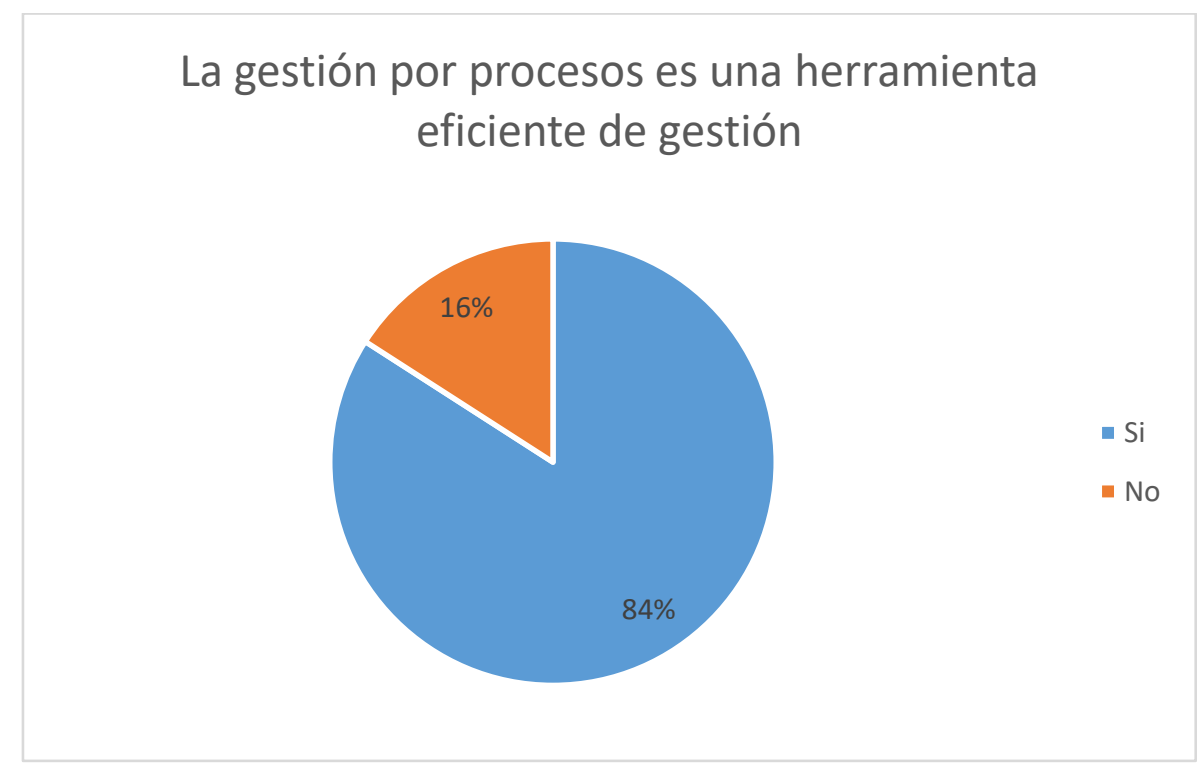

Gráfico 6. La gestión por procesos es una herramienta eficiente de gestión Fuente: Elaboración propia

\section{Interpretación}

Del total de encuestados, el $84 \%$ manifiestan que la gestión por procesos si es una herramienta eficiente de gestión, y el $16 \%$ creen que la gestión por procesos no es una herramienta eficiente de gestión, por lo expuesto, según la percepción de los ejecutivos este modelo si es una herramienta eficiente en la gestión de las empresas. 


\section{5. ¿La gestión por procesos es un sistema de control eficiente?}

\section{La gestión por procesos un sistema de} control eficiente

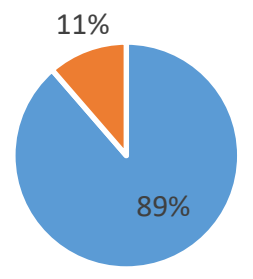

Gráfico 7. La gestión por procesos es un sistema de control eficiente Fuente: Elaboración propia

\section{Interpretación}

La percepción de los ejecutivos con respecto a esta pregunta, en un $89 \%$ consideran que la gestión por procesos si es un sistema de control eficiente en las empresas, y el $11 \%$ manifiestan lo contrario, por lo expuesto se puede evidenciar que este modelo si es un sistema de control eficiente en las empresas, ya que en la planificación se establecen metas y responsables en cada uno de los procesos, lo que facilita el seguimiento, la evaluación, el control y el mejoramiento continuo.

\section{CONCLUSIONES.}

- Según la percepción de los ejecutivos, el 89\% confirma que la gestión por procesos es un sistema de control eficiente en las empresas, ya que están entrelazadas las etapas de planificación, ejecución y control, lo que facilita el seguimiento, y la evaluación, por lo tanto este sistema, permite administrar los recursos de una empresa de manera eficiente, ya que disminuye el despilfarro y mejora los tiempos de operación, es decir aumenta la productividad y competitividad, mejora de la calidad y mejora el posicionamiento en el mercado, lo que coadyuva al incremento de los ingresos y de la rentabilidad.

- Es primordial que todas las personas que forman parte de la organización tengan como parte de sus objetivos la mejora continua de cada uno de los procesos que ejecutan y de los cuales forman parte, los colaboradores forman parte esencial del éxito en la mejora de los procesos pues ellos diariamente con la ejecución de los procesos, con la recepción de reclamos, con el comportamiento de los indicadores pueden identificar que procesos requieren mejora y proponer los cambios a realizar o ejecutarlos si esto se encuentra dentro de su alcance. 


\section{Referencias bibliográficas.}

Abascal, I. G. (2005). Análisis de Encuestas. Madrid: Esic.

Agudelo, T. L.F y Escobar, B, J. (2008). Gestion por Procesos. Bogotá: Incotec Internacional.

Agudelo, L. F. (2012). Evolucion de la Gestion por Procesos. Bogotá: Incotec Internacional.

Beltrán, et al. (2002). Guia para una Gestión Basada en Procesos. Madrid: Berekintza.

Bravo, J. B. (2009). Gestión de Procesos (Con responsabilidad Social). Santiago Chile: Evolución.

Bravo, J. C. (2011). Gestion de Procesos. $4^{\mathrm{a}}$ ed. Santiago de Chile: Evolución.

Consejo Nacional de Evaluación de la Política de Desarrollo Social. (2013). Manual para el diseño y la construcción de indicadores Intrumentos principales para el monitoreo de programas sociales de México. México DF: Coneval.

Espinel, V. A. (2013). Registro Oficial Organo del Gobierno del Ecuador. Quito: Secretaria Nacional de la Administración Pública .

Fomento, M. D. (2005). Gestion por Procesos - Modelos para implementar la mejorar continua en la Gestion de Empresas de transporte por Carretera.Madrid: Ministerio de Fomento.

Ogalla, S. F. (2005). Sistema de Gestión una guía práctica. Madrid: Diaz de Santos.

García, R. F. (2006). Sistema de Gestión de la Calidad, Ambiente y Prevencion de Riesgos Laborales. Su integración. Madrid: Gamma.

Gibson, J. et al. (2011). Las Organizaciones: Comportamiento, Estructura y Procesos. México D F: Edamsa Impresiones.

Larios, J. J. (2003). ISO 9001:2000 Guía práctica de normas para implantarlas en la empresa. México: Trillas.

Lemos, P. L. (2015). Como documentar un sistema de Gestión de calidad segun ISO 9001:2015. Madrid: Fundación Confemetal.

Lorea, M. V. (2006). Gestión del Cambio y la Innovación en la Empresa. Madrid: Ideaspropias.

Pacheco, et al. (2004). Indicadores Integrales de Gestión. Bogotá: Mc Graw Hill. 
Munch, et al. (2011). Organización diseño de estructuras organizacionales de alto rendimiento. México D F: Trillas.

Segura, F. O. (2005). Sistema de Gestion una guía Práctica. Madrid: Díaz de Santos.

Toro, R. (2015). Traducción Oficial Norma Internacional ISO 9001:2015. Sistemas De Gestion De La Calidad - Requisitos. SUIZA: Aenor.

Pérez de Fernández, J. V. (2004). Gestión por Procesos como utulizar ISO 9001:2000. Madrid: Esic.

Pérez de Fernández, J. V. (2010). Gestión por Procesos .4ª ed.Madrid: Esic.

Chango, P. R. (2015). Diseño y propuesta de un sistema de mejoramiento de los procesos del area de operaciones en plaza de las americas de la Cuidad de Quito. Quito; Escuela Politécnica Nacional

Silva, A. M. (2010). Teoria De Indicadores de Gestión y su Aplicacion Práctica. Bogotá; Universidad Militar Nueva Granada.

Champagnat, U. d. (2002). Gestión por Procesos. Recuperado el 11 de Enero del 2017, de Gestiopolis: http://www.gestiopolis.com/gestion-por-procesos/

Fernández, M. F. (2011). Jeraquia de Procesos. Recuperado el 8 de Mayo de 2017, de mi aula: https://miaula.blogia.com/2011/052303-jerarquia-de-procesos.php

Vergara, G. (2009). Sistema de Gestión. Recuperado el 31 de Marzo del 2019, de Mejora tu gestión: http://mejoratugestion.com/mejora-tu-gestion/que-es-un-sistema-degestion.

Zaratiegui, J. R. (1999). Economia Industrial. Recuperado el 31 de Mazo del 2016, de http://www.minetur.gob.es/Publicaciones/Publicacionesperiodicas/EconomiaIndustr $\mathrm{ial} /$ RevistaEconomiaIndustrial/330/12jrza.pdf.

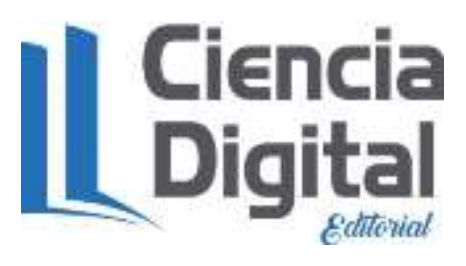




\section{PARA CITAR EL ARTÍCULO INDEXADO.}

Torres Guananga, G., Rodríguez Leon, J., Inca Falconi, A., Castelo Salazar, Ángel, \& Ríos Sanipatin, E. (2019). La gestión por procesos un sistema de control eficiente en las empresas. Ciencia Digital, 3(2.6), 495-514. https://doi.org/10.33262/cienciadigital.v3i2.6.600

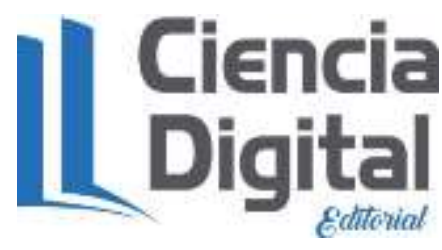

El artículo que se publica es de exclusiva responsabilidad de los autores y no necesariamente reflejan el pensamiento de la Revista Ciencia Digital.

El artículo queda en propiedad de la revista y, por tanto, su publicación parcial y/o total en otro medio tiene que ser autorizado por el director de la Revista Ciencia Digital.
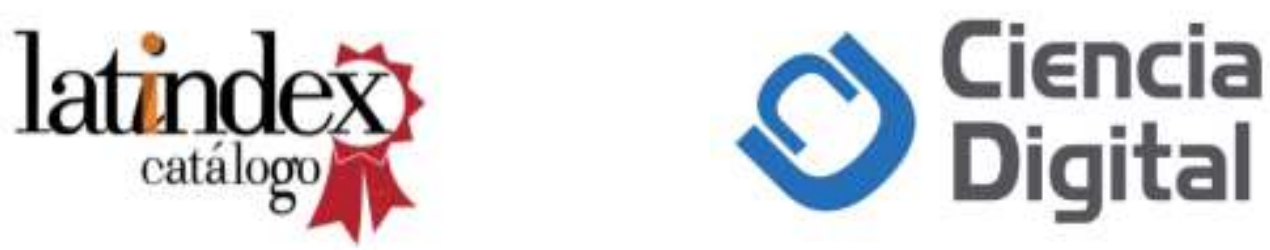